\title{
Nanocomposite Microcontainers
}

\author{
Christine M. Andres, Ińigo Larraza, Teresa Corrales, and Nicholas A. Kotov**
}

Microcontainers have many applications in smart packaging for pharmaceuticals, electronics, biological materials, and food products. They can be used as microreactors for expensive or media-sensitive components, or used to encapsulate chemical agents, cells, particles, etc. to prevent premature reactions based on a carefully controlled flux. ${ }^{[1,2]}$ This property also allows for their use as sensors based on selective permeation of components through the sidewalls. Arrayed microcontainers can aid in high-throughput analysis and fast optimization of component concentrations, sensing protocols, or (drug) delivery conditions. The fabrication of the majority of $3 \mathrm{D}$ microcontainers is currently achieved using complex, multistep, 2D lithographic processes predominantly using silicon or plastics. More advanced methods include elements of self-assembly where patterns are fabricated on planar surfaces and upon release are folded along pre-patterned hinges based on specific stimuli. ${ }^{[3-5]}$ Some methods of encapsulation also use templates made with molds or stamps fabricated by lithography. ${ }^{[2,6]}$ These variations of $2 \mathrm{D}$ lithography allow for variable micron scale porosity, ${ }^{[1,7]}$ while subnanometer-sized pores have been etched into the lids of some microcontainers by nanoimpriniting. ${ }^{[8]}$ Such structures are generally geared toward the encapsulation of cells for therapeutic purposes and have high perfusion rates. Microcontainers with slow release rates are desirable for implantable drug carriers and microreactors. Beyond controlled porosity and mechanical properties, microcontainers also often need transparency at different electromagnetic frequencies, for example for high-throughput analysis. ${ }^{[9]}$

While quite exciting and still under intense development, the current methods to make microcontainers have two significant limitations:

1) the choice of materials available for sidewalls and other parts of microcontainers and therefore the range of transport, optical, mechanical, and biological properties available is quite limited; and

2) the choice of microcontainer contents is strongly restricted by chemicals/solvents or temperatures used during fabrication/assembly and by the limited compatibility between the solvents used for loading and the walls of the microcontainer.

C. M. Andres, Prof. N. A. Kotov

Department of Chemical Engineering

University of Michigan

2300 Hayward St, Ann Arbor, MI 48109, USA

E-mail:kotov@umich.edu

I. Larraza, Prof. T. Corrales

Polymer Photochemistry Group

Instituto de Ciencia y Tecnologia de Polimeros

3 Juan de la Cierva, Madrid, 28006 Spain

DOI: 10.1002/adma.201201378
Both of these issues result in significant delays in the development of most applications mentioned above as well as substantially increase the cost of microcontainer fabrication, which also affects their potential, for instance, for high-throughput reaction optimization or sensing.

Here we demonstrate a methodology for microcontainers to be made from nanocomposites rather than silicon or PDMS. The technique can be applicable to a very broad range of polymer-polymer or polymer-nanoparticle combinations. Considering the problems mentioned above, nanocomposites offer unique combinations and an exceptionally wide range of chemical and physical properties that can be easily adapted to specific applications. All-nanocomposite microcontainers can provide inert packaging with slow/controlled release for virtually any type of encapsulating material. They also can be made in high yield and inexpensively using layer-by-layer assembled (LBL) multilayers with 3D patterns. Among the many techniques to fabricate nanocomposites, $\mathrm{LBL}^{[10]}$ stands out due to the universality, simplicity and unlimited combinations of remarkable material properties possible by the thermodynamically limited assembly of high-molecular-weight materials ${ }^{[11-17]}$ and high performance. ${ }^{[18,19]}$ The well-known ability of the LBL technique to make controlled-release coatings with stratified spatially separated reagents ${ }^{[20-22]}$ and spherical microcapsules. ${ }^{[23,24]}$ adds to the versatility of the method. At the same time, nanocomposite microcontainers, especially in arrayed form, address different technological challenges than these embodiments of the LBL technique.

LBL nanocomposites from polyvinyl alcohol (PVA) and sodium montmorillonite clay nanoplatelets (MTM) present a particularly attractive nanomaterial for microcontainer walls due to its low permeability of oxygen particularly suitable for drug carriers, ${ }^{[25]}$ and its high optical transparency, flexibility, and tensile strength ${ }^{[26,27]}$ attributed to controlled orientation of the anisotropic clay nanoplatelets. Note that (PVA/MTM) multilayers also have high biocompatibility, ${ }^{[28]}$ and are resistant to both hydrophilic and hydrophobic solvents. All together this prompted us to demonstrate the conceptual possibility of fabricating arrays of well-and-lid microcontainers from this nanocomposite despite limited knowledge of methods to integrate LBL nanocomposites and microtechnologies. ${ }^{[29]}$ To generate 3D patterns from a traditionally planar material, we used a template approach with a poly(dimethylsiloxane) (PDMS) mold (Scheme 1). Initially, a $1 \mathrm{~cm}$ thick layer of PDMS was cured onto a silicon wafer with patterned $0.18 \mu \mathrm{L}$ capacity microwells $(3 \mathrm{~mm} \times 3 \mathrm{~mm} \times 20 \mu \mathrm{m})$ to create a PDMS mold with microtowers (Figure 1a). (PVA/MTM) ${ }_{n}$ multilayers, where $n$ is the number of LBL deposition cycles, were deposited onto the released PDMS microtower mold and a planar PDMS sheet to make the wells and the lids of the microcontainers, respectively. Due to the conformal and self-limiting nature of LBL assembly, the film takes on the shape of the PDMS microwell 


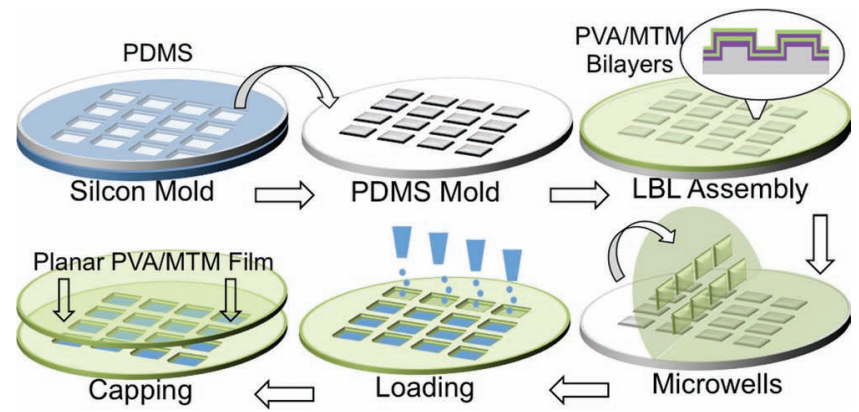

Scheme 1. A PDMS mold with microtowers is fabricated from a silicon mold. Alternate deposition of the PDMS mold into PVA and MTM with intermediate rinsing and drying steps, results in the growth of an LBL assembled film onto the surface. This film is then peeled off to reveal nanocomposite microwells that can be loaded by micropipetting. After loading, a planar LBL film is then pressed on top of the microwells to form a lid sealed by hydrogen bonding.

template and does not fill in the cavities as would be expected for other methods of thin film fabrication, i.e., spin-coating or blade-spreading (Figure 1b,c). This results in a 3D pattern in the LBL film without the need for additional processing. Importantly, the (PVA/MTM) 1000 film maintains the layered structure (Figure 1d) that is often granted responsibility for the excellent mechanical, optical and barrier properties of the material.[27] The same layered structure, especially for the "lid" of the microcontainer, will also offer unique control over gas/liquid permeability. As such, a lid was made from (PVA/MTM) ${ }_{1000}$, however its thickness and composition can be varied widely to regulate, for instance permeation and/or response to external stimuli, e.g., magnetic field. ${ }^{[30]}$ The assembly of the microcontainer array was achieved by placing the planar (PVA/MTM) $)_{1000}$ sheet on to the (PVA/MTM) ${ }_{1000}$ sheet with patterned wells followed by application of pressure onto the back of the planar substrate to allow for hydrogen bonding to occur between the PVA/MTM multilayers of each sheet and a sealed microcontainer to be fabricated (Figure 2a,b). Mechanical properties of the (PVA/ MTM $)_{1000}$ allow this transfer to occur with ease. Previously microchambers have been fabricated from the LBL assembly of polyelectrolytes, ${ }^{[31,32]}$ however having a Young's modulus, E, of $\sim 4 \mathrm{GPa}$ and other mechanical properties substantially weaker than LBL clay nanocomposites with $E=125 \mathrm{GPa}^{[26]}$ can be quite restrictive. For instance, they were reported to exist only while being supported by a solid substrate. Also while loading has not been demonstrated with these chambers, it is likely due to the mechanical properties that solvent-exchange methods would be required, as is done in many cases with microcapsules. ${ }^{[16]}$

In addition to the fabrication of the microcontainer itself, it is important to consider the methodology of its loading. To further investigate the nanocomposite microcontainers as an advanced packaging technology, trioctylphosphine oxide (TOPO) stabilized CdSe nanoparticles (NPs) or citrate-stabilized gold NPs, synthesized according to previously published procedures, ${ }^{[33,34]}$ were loaded into the microwells by microinjection (Figure 2c) before the lid was sealed. The microinjection loading procedure used here does not rely on self-loading equilibrium conditions or special thermal or chemical treatments and allows for a wide variety of materials to be loaded into the microcontainers. It can also be integrated with inkjet technology, ${ }^{[35]}$ allowing for controlled mixtures, live cells, or even reacting species to be loaded into the nanocomposite microcontainers.

The fluorescence of the Cd-Se NPs was clearly observed under UV-light through the transparent nanocomposite walls (Figure 3a). The robustness of the microcontainers was tested by exposure of an array of six sealed microcontainers to chloroform, a solvent capable of dispersing the TOPO-stabilized NPs. After soaking for several hours, the fluorescence of the NPs loaded into the microcontainers submerged in chloroform was observed to be localized exclusively in the original wells, suggesting that they are well protected and sealed (Figure $3 \mathrm{~b}$ ). The walls of the nanocomposite container were not compromised nor any detectable release of NPs observed after the sealed microcontainers were placed in vials filled with water, ethanol, or acetone. Similarly, when the microcontainers were loaded with citrate stabilized gold NPs, a water dispersible nanomaterial, and submerged in water for $24 \mathrm{~h}$, the NPs remained localized in the well of the microcontainer (Figure 3c) suggesting the ability of the microcontainers to retain a variety of different materials in numerous solvents.

Beyond clay nanocomposites, LBL offers a wide variety of materials that can be incorporated into the walls of the microcontainer as the technique is not limited by lithographic processing of the material or special conditions required for
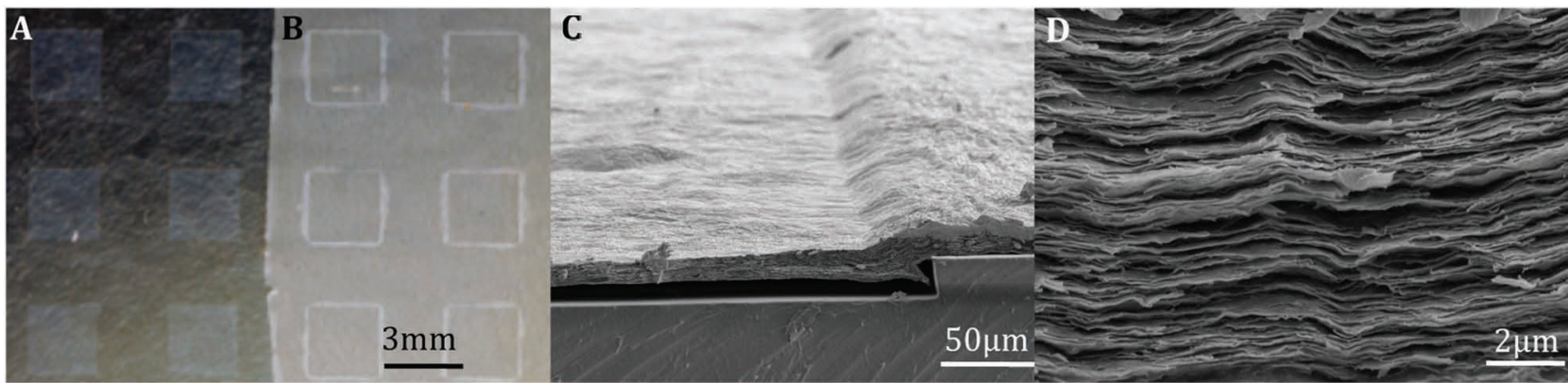

Figure 1. A) Photograph of PDMS mold with microwell shape. B) Clay nanocomposite film deposited on PDMS template with LBL assembly. C) SEM image of clay nanocomposite LBL film with the contour of the PDMS microwell. D) SEM image of the layered structure of the LBL clay nanocomposite that contributes to the unique properties of the material that are advantageous for microcontainer walls. 

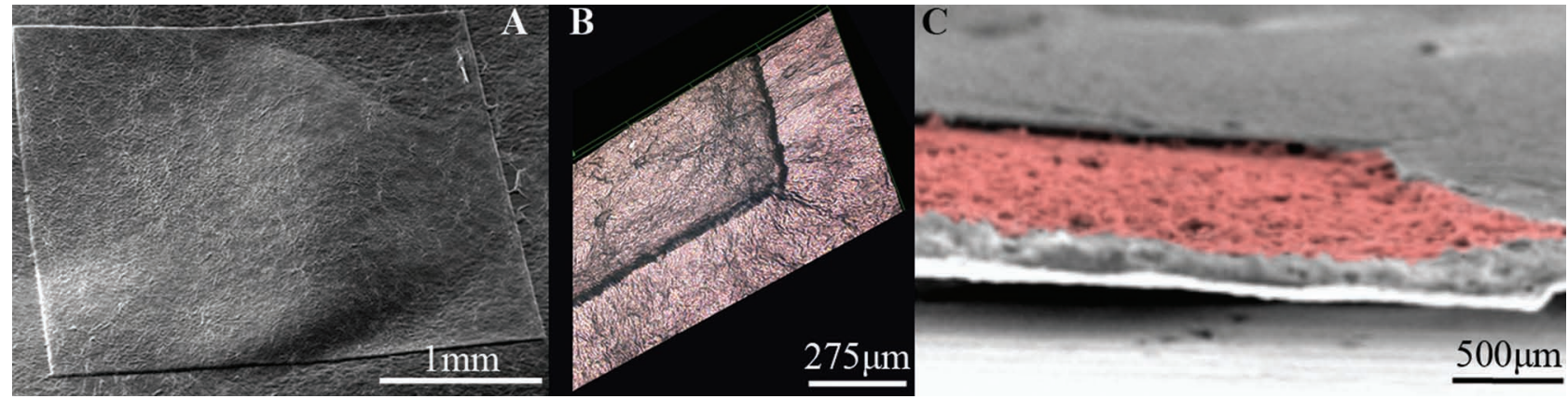

Figure 2. A) SEM image and B) 3D confocal microscopy image of capped clay nanocomposite microcontainer. C) SEM image of micro-well filled with CdSe NPs (the color has been artificially added).

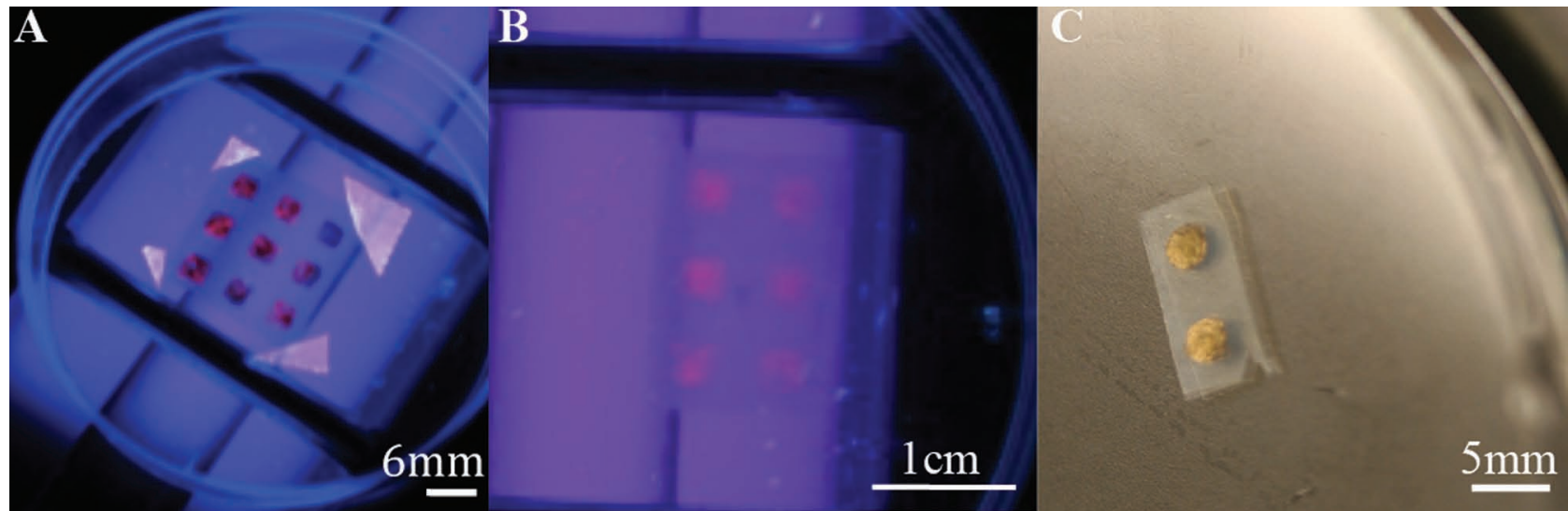

Figure 3. A) Photograph of nine CdSe-filled microcontainers in the presence of UV light demonstrating transparency to optical and ultraviolet wavelengths. Photograph of clay nanocomposite microcontainer filled with B) CdSe NPs under UV-light in the presence of chloroform and C) gold NPs in the presence of water

folding. The strict control over material stratification provided by LBL allows for control over the interior and exterior surface properties of the microcontainer as well as for the simple incorporation of other materials into the MTM nanocomposite such as silver NPs for antimicrobial action, ${ }^{[36]}$ magnetite NPs ${ }^{[37]}$ or the growth of carbon nanotubes within LBL assembled films. ${ }^{[38]}$ The simple, low-cost, and universal LBL deposition operates at low temperature and mild conditions outside of a clean room, providing a sustainable option for universal microcontainers.

\section{Supporting Information}

Supporting Information is available from the Wiley Online Library or from the author.

\section{Acknowledgements}

This material is based upon work partially supported by the Center for Solar and Thermal Energy Conversion, an Energy Frontier Research Center funded by the U.S. Department of Energy, Office of Science, Office of Basic Energy Sciences under Award Number \#DE-SC0000957.
We acknowledge support from the NSF under grant ECS-0601345; EFRIBSBA 0938019; CBET 0933384; CBET 0932823; CBET 1036672; and a Graduate Research Fellowship. The work is also partially supported by AFOSR MURI 444286-P061716, DARPA MATLOG program, NIH 1R21CA121841-01A2, and MICINN (Spain) under MAT2009-09671. The authors would like to thank Dr. Pilar Herrera-Fierro and the Lurie Nanofabrication Facility for preparation of the silicon wafer, the University of Michigan's EMAL for its assistance with electron microscopy, and Dr. Daniel Lilly for his support with CdSe nanoparticle synthesis.

Received: April 4, 2012

Revised: April 12, 2012

Published online: June 25, 2012

[1] C. L. Randall, T. G. Leong, N. Bassik, D. H. Gracias, Adv. Drug Deliv. Rev. 2007, 59, 1547.

[2] T. G. Leong, A. M. Zarafshar, D. H. Gracias, Small 2010, 6, 792.

[3] R. R. A. Syms, E. M. Yeatman, V. M. Bright, G. M. Whitesides, J. Microelectromech. Syst. 2003, 12, 387.

[4] T. Leong, Z. Y. Gu, T. Koh, D. H. Gracias, J. Am. Chem. Soc. 2006 128, 11336.

[5] L. Ionov, Soft Matter 2011, 7, 6786.

[6] J. G. Fernandez, A. Khademhosseini, Adv. Mater. 2010, 22, 2538. 
[7] C. L. Randall, Y. V. Kalinin, M. Jamal, T. Manohar, D. H. Gracias, Lab Chip 2011, 11, 127.

[8] J. Kwon, K. Trivedi, N. V. Krishnamurthy, W. Hu, J. B. Lee, B. Gimi, J. Vac. Sci. Technol. B 2009, 27, 2795.

[9] A. Azam, K. E. Laflin, M. Jamal, R. Fernandes, D. H. Gracias, Biomed. Microdevices 2011, 13, 51.

[10] G. Decher, Science 1997, 277, 1232.

[11] P. T. Hammond, Adv. Mater. 2004, 16, 1271.

[12] Y. Wang, A. S. Angelatos, F. Caruso, Chem. Mater. 2008, 20, 848.

[13] F. Caruso, Adv. Mater. 2001, 13, 11.

[14] K. Ariga, J. P. Hill, Q. M. Ji, Phys. Chem. Chem. Phys. 2007, 9, 2319.

[15] J. F. Quinn, A. P. R. Johnston, G. K. Such, A. N. Zelikin, F. Caruso, Chem. Soc. Rev. 2007, 36, 707.

[16] C. S. Peyratout, L. Dahne, Angew. Chem. Int. Edit. 2004, 43, 3762.

[17] X. Zhang, H. Chen, H. Y. Zhang, Chem. Commun. 2007, 1395.

[18] R. H. A. Ras, Y. Umemura, C. T. Johnston, A. Yamagishi, R. A. Schoonheydt, Phys. Chem. Chem. Phys. 2007, 9, 918.

[19] P. Podsiadlo, B. S. Shim, N. A. Kotov, Coord. Chem. Rev. 2009, 253, 2835.

[20] J. Zhang, L. S. Chua, D. M. Lynn, Langmuir 2004, 20, 8015.

[21] A. J. Chung, M. F. Rubner, Langmuir 2002, 18, 1176.

[22] K. C. Wood, N. S. Zacharia, D. J. Schmidt, S. N. Wrightman, B. J. Andaya, P. T. Hammond, Proc. Natl. Acad. Sci. USA 2008, 105, 2280.

[23] F. Caruso, R. A. Caruso, H. Mohwald, Science 1998, 282, 1111.
[24] G. B. Sukhorukov, A. A. Antipov, A. Voigt, E. Donath, H. Möhwald, Macromol. Rapid Commun. 2001, 22, 44

[25] W. S. Jang, I. Rawson, J. C. Grunlan, Thin Solid Films 2008, 516, 4819.

[26] P. Podsiadlo, A. K. Kaushik, E. M. Arruda, A. M. Waas, B. S. Shim, J. D. Xu, H. Nandivada, B. G. Pumplin, J. Lahann, A. Ramamoorthy, N. A. Kotov, Science 2007, 318, 80.

[27] Z. Tang, Nat. Mater. 2003, 2, 413.

[28] F. Hua, T. Cui, Y. M. Lvov, Nano Lett. 2004, 4, 823.

[29] K. Ariga, M. McShane, Y. M. Lvov, Q. M. Ji, J. P. Hill, Expert Opin. Drug Deliv. 2011, 8, 633.

[30] A. Mamedov, J. Ostrander, F. Aliev, N. A. Kotov, Langmuir 2000, 16, 3941.

[31] M. V. Kiryukhin, S. M. Man, S. R. Gorelik, G. S. Subramanian, H. Y. Low, G. B. Sukhorukov, Soft Matter 2011, 7, 6550.

[32] M. V. Kiryukhin, S. M. Man, A. Tonoyan, H. Y. Low, G. B. Sukhorukov, Langmuir 2012, 28, 5678.

[33] D. V. Talapin, A. L. Rogach, A. Kornowski, M. Haase, H. Weller, Nano Lett. 2001, 1, 207.

[34] J. Turkevich, P. C. Stevenson, J. Hillier, Disc. Faraday Soc. 1951, 55.

[35] C. M. Andres, N. A. Kotov, J. Am. Chem. Soc. 2010, 132, 14496.

[36] P. Podsiadlo, S. Paternel, J. M. Rouillard, Z. F. Zhang, J. Lee, J. W. Lee, L. Gulari, N. A. Kotov, Langmuir 2005, 21, 11915.

[37] A. A. Mamedov, N. A. Kotov, Langmuir 2000, 16, 5530.

[38] J. Li, S. Srivastava, J. Ok, Y. Zhang, M. Bedewy, N. Kotov, Chem. Mater. 2011, 23, 1023. 